\title{
Pericyazine in the treatment of cannabis dependence in general practice: a naturalistic pilot trial
}

This article was published in the following Dove Press journal:

Substance Abuse and Rehabilitation

25 May 2012

Number of times this article has been viewed

\author{
Kirsten C Morley' \\ Paul S Haber ${ }^{1,2}$ \\ Madeleine L Morgan ${ }^{3}$ \\ Fares Samara ${ }^{3,4}$ \\ 'Discipline of Addiction Medicine, \\ Sydney Medical School, University \\ of Sydney, NSW, Australia; ${ }^{2}$ Drug \\ Health Services, Royal Prince Alfred \\ Hospital, Camperdown, NSW, \\ Australia; ${ }^{3}$ Drug and Alcohol Services, \\ North Coast Area Health Service, \\ Kempsey and Port Macquarie, NSW, \\ Australia; ${ }^{4}$ Durri Aboriginal Medical \\ Service, Kempsey, NSW, Australia
}

Correspondence: Fares Samara

Drug and Alcohol Services, North Coast Area Health Service, Kempsey and Port Macquarie, NSW, Australia

Email fares.samara@ncahs.health.nsw. gov.au

\begin{abstract}
Cannabis is one of the most widely used illicit drugs worldwide. However, while the rates of cannabis dependence and treatment increase, there remains no medications approved for this use. Due to its sedative effects and low abuse liability, the typical antipsychotic pericyazine has been utilized in some parts of Australia for the treatment of cannabis dependence. We aimed to provide documentation of preliminary outcomes and acceptability of pericyazine treatment in a small sample. A naturalistic case series study was conducted in which 21 patients were enrolled for a 4-week course of pericyazine (up to $8 \times 2.5 \mathrm{mg}$ tablets daily) and weekly medical review. Levels of cannabis use were reported and side effects with electrocardiography and blood tests were monitored. Measures of dependence severity, depression, anxiety, and insomnia were taken at baseline and follow-up utilizing validated psychometric tools. Significant reductions in cannabis use, depression, anxiety, and insomnia severity occurred across time. Pericyazine appeared to be well tolerated and easily administered in the community clinics. The results provide some preliminary evidence that low-dose short-term pericyazine may be an acceptable mode of treatment in this population. Given the open-label nature of the design, we cannot conclude that pharmacotherapy was uniquely responsible for the treatment effect. Nonetheless, low-dose pericyazine may be a potentially effective approach to the treatment of cannabis dependence, and further evaluation via a randomized placebo-controlled trial is warranted.
\end{abstract}

Keywords: cannabis, antipsychotic, pharmacotherapy, addiction, pericyazine

\section{Introduction}

Cannabis is the most commonly used illicit drug in the world, particularly in Australia, New Zealand, and the United States. ${ }^{1}$ Thus, although the dependence liability of cannabis is lower than that of other illegal substances, such as heroin, ${ }^{2}$ there exists a substantial number of individuals who develop cannabis use disorders. ${ }^{3}$ The rate of treatment seeking for lifetime cannabis abuse and dependence is on the increase, with cannabis accounting for $27 \%$ of closed treatment episodes annually. ${ }^{3}$ Treatmentseeking cannabis users report distress with regards to their use ${ }^{4}$ and, alarmingly, relapse rates of up to $90 \%$ have been observed..$^{5}$ Withdrawal symptoms such as craving, anxiety, agitation, and sleep disturbances, have been found to significantly contribute to relapse. ${ }^{3,6,7}$ This illuminates the importance of developing pharmacotherapies targeted at these symptoms for individuals who may be less responsive to other forms of treatments.

However, there are no medications currently approved for the treatment of cannabis dependence, and there exists a paucity of research investigating effective pharmacotherapies. ${ }^{8}$ Some agents have been investigated and yielded either little 
clinical efficacy or lack controlled comparisons, such as the mood stabilizer divalproex and lithium ${ }^{9,10}$ and the antidepressants bupropion and nefazodone. ${ }^{11,12}$ The oral cannabinoid agonist $\Delta$ 9-tetrahydrocannabinol (THC) has been reported to decrease a number of withdrawal symptoms such as craving, ${ }^{9,13}$ but has shown little effect on sleep disturbance, irritability, and decreasing relapse to cannabis use. ${ }^{14,15}$ Moreover, the majority of these studies have been limited to the controlled environment of inpatient settings or have not employed cannabis-dependent treatment seekers. As such, the feasibility of an agonist substitution strategy within the target community is yet to be ascertained.

There is emerging evidence to suggest that antipsychotic medication may be helpful in the treatment of substance abuse. The rationale is that during the period of detoxification, patients with substance abuse disorders often develop psychiatric symptoms, including insomnia, depression, anxiety, and psychosis. ${ }^{16,17}$ In addition, antipsychotic drugs possess relatively low abuse liability given their antagonism of $\mathrm{D}^{2}$ receptors that mediate reward processes. ${ }^{18}$ The atypical antipsychotic quetiapine has been noted to have some efficacy, ${ }^{19}$ with a case report and a recent open-label study observing substantial reductions in cannabis use. ${ }^{20,21}$ Quetiapine was found to reduce cannabis use by $97 \%$ over a 6-month period in four schizophrenic patients and four patients with borderline personality disorder. ${ }^{20}$ Nonetheless, this potent antipsychotic is indicated for the manifestation of psychosis and may not be readily accepted by the cannabis-dependent population in an outpatient setting.

Pericyazine is a mild typical antipsychotic that is used as an adjunct to the short-term management of severe anxiety states and psychosis ${ }^{22}$ and is listed on the Australian Pharmaceutical Benefits Scheme. Due to its low abuse liability, low cost, and an anxiolytic and sedative profile, pericyazine has been used in some parts of Australia for the treatment of cannabis dependence in patients with moderate to severe withdrawal symptoms. The agent has now been prescribed for 9 years in several clinics on the mid-north coast of New South Wales, Australia, to over 300 patients at the low dose of 10-20 mg/day for a median period of 4 weeks. In this area of health service, approximately $15 \%$ of intake is for cannabis treatment seekers who are most often male in the 30-39-year range, one-third indigenous, and with severe dependence. The current study aimed to provide a preliminary investigation into the efficacy of pericyazine in the treatment of cannabis dependence following these anecdotal reports.

\section{Methods}

\section{Participants}

Participants were 21 men and women who presented to one of three outpatient treatment services on the mid-north coast of New South Wales: Kemspey (Aboriginal Medical Service; Drug and Alcohol Service) or Port Macquarie (Drug and Alcohol Service). Inclusion criteria for the pericyazine group were: (i) age between 18 and 65 years, (ii) clinician-rated DSM-IV diagnosis of cannabis dependence, (iii) willingness to give informed consent, (iv) adequate cognition and English language skills. Exclusion criteria were (i) sensitivity to or treatment with antipsychotic therapy within 6 months, (ii) pregnancy or breastfeeding, (iii) coagulopathy, (iv) a long period (eg, $>7$ days) of abstinence from cannabis before treatment initiation, (v) severe psychiatric disorder associated with psychosis and significant suicide risk. Stable mental health or methadone maintenance clients were considered if they met the inclusion criteria. All participants provided written informed consent. The study was approved by the Human Ethics Review Committee of the North Coast Area Health Service.

\section{Procedure}

Patients received a 4-week regime of pericyazine, which consisted of up to $8 \times 2.5 \mathrm{mg}$ tablets per day as one tablet every 3 hours and two tablets at night for 2 weeks, then up to six tablets per day for the following week and up to four tablets per day for the last week. However, this regime was monitored and altered in response to withdrawal symptoms and/or side effects. For example, the night-time dose would be increased in the case of vivid nightmares, while the day-time dose decreased in the case of tiredness. In some cases, cannabis use was gradually reduced and the dose of pericyazine was titrated such that the dose regime increased up to the maximum of $8 \times 2.5 \mathrm{mg}$ tablets per day as patients became abstinent. In most cases medication was dispensed on a weekly basis but in a small number of cases where individual risk assessment indicated, it was dispensed daily or near daily.

\section{Clinical assessments}

All participants received one medical assessment (baseline) and two to four reviews, including the 4-week follow-up appointment. At the initial baseline medical assessment, cannabis use in the previous 30 days (cones per day/week), years of cannabis use, abstinence history, previous withdrawal experience, method of ingestion, and other drug use was determined. At each appointment a blood sample was 
obtained to test for full blood count (FBC), liver function test (LFT), electrolytes/urea/creatinine (EUC), and thyroidstimulating hormone (TSH). Electrocardiography (ECG) was also obtained. Recent cannabis use and compliance were assessed through self-reporting. Clinical ratings performed at baseline and the 4-week follow-up included the Severity of Dependence Scale (SDS), ${ }^{23}$ Insomnia Severity Index (ISI), ${ }^{24}$ and Depression Anxiety Stress Scale (DASS 21-item). ${ }^{25}$ Side-effects and withdrawal symptoms were reported by each patient when experienced and recorded by the clinician.

\section{Statistical analyses}

Only patients that provided confirmation of taking at least one dose of the medication were included in the outcome analyses. The primary outcome analysis was an intention-to-treat mixed model for repeated measurements of cannabis use (cones per day) across time (five time points, weeks 0-4). Repeated-measures analysis of variance was employed to observe the effect of time on SDS, DASS, and ISI scores. All analyses were with significance level at $P<0.05$. Data were analyzed using SPSS software (v 18 for Macintosh; SPSS Inc, Chicago, IL).

\section{Results}

Twenty-one patients were enrolled in the current study. Baseline characteristics are depicted in Table 1. The

Table I Intention-to-treat baseline characteristics of the study population

\begin{tabular}{|c|c|}
\hline & Pericyazine $(n=21)$ \\
\hline \multicolumn{2}{|l|}{ Demographics } \\
\hline Age & 31.1 \\
\hline Male, $\%$ & 71 \\
\hline Employed, \% & 42 \\
\hline Married or defacto, $\%$ & 40 \\
\hline Aboriginality, \% & 24 \\
\hline \multicolumn{2}{|l|}{ Cannabis use } \\
\hline Cannabis in last 30 days, $\%$ & 100 \\
\hline Cones/day & $28.4 \pm 17.1$ \\
\hline Cones/week & $204.0 \pm 119.6$ \\
\hline Tobacco in cones, $\%$ & 95 \\
\hline Age of first use & $14.1 \pm 2.3$ \\
\hline Years of negative affect & $10.3 \pm 8.4$ \\
\hline \multicolumn{2}{|c|}{ Alcohol and other drug use in last 30 days } \\
\hline Alcohol, \% (n) & 91 (19) \\
\hline Alcohol daily, \% (n) & $29(6)$ \\
\hline Alcohol weekly, \% (n) & $43(9)$ \\
\hline Methadone program, \% (n) & $5(I)$ \\
\hline Cocaine, $\%(n)$ & $5(I)$ \\
\hline Amphetamines, \% (n) & $10(2)$ \\
\hline Cigarettes per day & $17.8 \pm 8.0$ \\
\hline
\end{tabular}

Notes: Data represent mean \pm standard deviation unless otherwise stated. There were no significant differences between groups. Amphetamine and cocaine use was reported to be infrequent within the previous 30 days (once or twice). average age was 31 years, 71\% were male, $40 \%$ married, $42 \%$ employed, and 24\% were Aboriginal or Torres Strait Islander. The mean (median) number of cones smoked was 28.4 (20) per day and 204.0 (175) per week, whereby 95\% of participants mixed their cannabis with tobacco and almost all participants smoked waterpipes. The sample reported approximately 10 years of negative effects of cannabis use. Of the total, 91\% consumed alcohol in the past month and $43 \%$ daily. For scores on the SDS or cannabis use, no participants fell under the adult cut-off range of $3 .{ }^{26}$

Out of these, 19 confirmed taking at least one dose of the medication and six had dropped out by week 4 (28\%). All tests for FBC, ECG, EUC, and TSH were within the normal range at baseline and follow-up. The most common side effects were drowsiness $(21 \%)$, headaches $(5 \%)$, and rigidity/twitching (5\%). These were alleviated with a reduction of daytime dosage on a case-by-case basis. No patients discontinued treatment due to side effects, and there were no reported adverse events. The average (standard deviation) daily dose of pericyazine (Table 2 ) was $15.26 \mathrm{mg}( \pm 4.40)$ for week $1,13.91 \mathrm{mg}( \pm 4.56)$ for week $2,11.5 \mathrm{mg}( \pm 5.81)$ for week 3 , and $8.16 \mathrm{mg}( \pm 6.78)$ for week 4 . There was no evidence of overdose or abuse of pericyazine. Withdrawal symptoms that were reported included depression $(5 \%)$, sweating (16\%), irritability (5\%), sleep difficulty $(26 \%)$, craving (12\%), anxiety (11\%), aggression (5\%), and strange dreams $(16 \%)$.

Table 2 depicts the intention-to-treat pattern of cannabis use for patients that reported taking at least one dose of pericyazine $(n=19)$. An improvement over time in

Table 2 Change in baseline and follow-up psychological characteristics for participants treated with pericyazine $(n=15)$

\begin{tabular}{|c|c|c|}
\hline Psychological measure & Baseline & Follow-up \\
\hline Severity of Dependence & $9.8 \pm 3.7$ & $2.7 \pm 2.8$ \\
\hline Scale (SDS)/I5* & (dependent) & (not dependent) \\
\hline Insomnia Severity Index & $\mid 4.1 \pm 8.6$ & $3.5 \pm 4.9$ \\
\hline \multirow[t]{2}{*}{$(\mathrm{ISI}) / 28^{*}$} & (moderate & (no clinical levels \\
\hline & severity) & of insomnia) \\
\hline Depression & $24.0 \pm 11.6$ & $6.6 \pm 9.2$ \\
\hline \multirow[t]{2}{*}{$(\mathrm{DASS}) / 42 *$} & (severe) & (no clinical levels \\
\hline & & of depression) \\
\hline Anxiety & $16.8 \pm 11.7$ & $3.6 \pm 4.6$ \\
\hline \multirow[t]{2}{*}{ (DASS)/42* } & (severe) & (no clinically levels \\
\hline & & of anxiety) \\
\hline Stress & $24.9 \pm 10.8$ & $6.9 \pm 6.9$ \\
\hline \multirow[t]{2}{*}{ (DASS)/42* } & (moderate) & (no clinically levels \\
\hline & & of stress) \\
\hline
\end{tabular}

Notes: Data represent mean \pm standard deviation unless otherwise stated. $* P<0.00$ I, significant effect of time, repeated-measures analysis of variance. Abbreviations: SDS, Severity of Dependence; ISI, Insomnia Severity Index; DASS, Depression Anxiety Stress Scale. 
Table 3 Intention-to-treat analysis of self-reported number of cones smoked and dose of pericyazine per day

\begin{tabular}{llllll}
\hline & Baseline & Week I & Week 2 & Week 3 & Week 4 \\
\hline Cones smoked (per day)* & $28.0 \pm 17.5$ & $7.2 \pm 8.2$ & $2.1 \pm 3.3$ & $1.1 \pm 1.6$ & $0.8 \pm 1.3$ \\
Pericyazine (mg per day) & $\mathrm{n} / \mathrm{a}$ & $15.3 \pm 4.4$ & $13.9 \pm 4.6$ & $11.5 \pm 5.8$ & $8.2 \pm 6.8$ \\
\hline
\end{tabular}

Notes: Data represent mean \pm standard deviation for 19 out of 21 enrolled patients who confirmed taking at least one dose of medication. $*$ Mixed models indicated there was a significant effect of time for cones smoked per day $\left(\mathrm{F}_{4,18}=14.39 ; P<0.000 \mathrm{I}\right)$.

cannabis use levels was demonstrated smoking on average one cone per day [seven cones per week] following treatment compared to 28 cones per day [196 cones per week] at baseline $\left[\mathrm{F}_{4,18}=14.39, P<0.0001\right]$. At follow-up $42 \%$ of the sample were abstinent. Throughout the treatment period, approximately $50 \%$ gradually tapered their use to abstinence or reduced their use to less than $5 \%$ of their reported use at baseline, while approximately $20 \%$ abruptly ceased smoking (abstinence) or reduced their use to less than $5 \%$ of their reported use at baseline. No patient showed an increase in tobacco smoking, one quit smoking (from 15 cigarettes/day), and two stated they increased alcohol consumption. There were no reports of increased use in other drugs of abuse or changes in methadone dose on the maintenance program.

Mean scores of psychological measures at baseline and follow-up are depicted in Table 3. Repeated measures revealed a significant effect of time between baseline and follow-up for all measures $(P<0.001$ for all).

\section{Discussion}

This is the first documentation of the acceptability, feasibility, and preliminary outcomes of pericyazine treatment for cannabis dependence. Over the 4-week trial period, participants displayed a significant reduction in cannabis use over time and a reduction in SDS scores from baseline. Direct comparisons between studies on cannabis dependence have been noted to be problematic because of wide variations in duration of treatment, study design, withdrawal measures, sample selection, and setting. ${ }^{10}$ Indeed, the current sample demonstrated somewhat higher baseline levels of cannabis use (mean of 28 cones per day) compared to other Australian studies with nonindigenous and indigenous samples. ${ }^{10,27,28}$ These discrepancies are possibly due to variations in the potency of cannabis and the mixing of tobacco. Nonetheless, the mean baseline SDS scores across studies are similar and the change in SDS following pericyazine treatment is comparable to a recent inpatient open-label study of lithium and cannabis withdrawal. ${ }^{10}$

In addition, significant reductions in DASS depression levels and the ISI were observed. Those treated with pericyazine demonstrated a 16-point reduction in DASS depression from the mean category at baseline being "severe" to "normal" at follow-up, suggesting a clinical meaningful change. Pericyazine-treated participants experienced an approximately 11-point reduction in ISI scores, which is encouraging considering that a six-point reduction has been recommended to represent a clinically meaningful improvement. ${ }^{29}$

Pericyazine was generally well tolerated among patients with no adverse events and no discontinuations due to side effects. Although attrition rates in treatment studies for cannabis dependence studies are not widely published, a dropout rate of $50 \%$ is quite common, ${ }^{30}$ such that our drop-out rate of $25 \%$ is within an acceptable range. The most common reported side effect was drowsiness, and this was sufficiently alleviated with a reduction of daytime dosage. Nonetheless, more information is required regarding the treatment efficacy and side-effect profile of pericyazine during the acute withdrawal phase. Withdrawal symptoms peak several days after last cannabis use, with some occurring within the first 2 weeks of abstinence; ${ }^{31}$ further research with strict monitoring of daily cannabis use, withdrawal, and side-effect data is required.

The current study has several limitations, including its small size, absence of an adequately matched control group, open-label design, and reliance on self-reporting. Open-label studies are subject to low internal validity due to potential confounders, such as clinician bias and expectancy bias. ${ }^{32}$ This study does have the advantage, however, of being implemented in a natural setting by the regular treating clinicians and thus provides "real life" data regarding a cannabis pharmacotherapy already adopted in the outpatient community. Notwithstanding, little can be definitely concluded about the efficacy of pericyazine independently from our results and a controlled trial is warranted.

\section{Conclusion}

This is the first documentation of the application of pericyazine in the treatment of cannabis dependence. The results provide some preliminary evidence that low-dose short-term pericyazine may be an acceptable mode of treatment in this population, although a randomized, double-blind, placebocontrolled trial is required. 


\section{Disclosure}

This study was funded by a grant from the Hastings Macleay General Practice Network (NSW, Australia). The authors have no conflicts of interest or sources of funding that may be relevant to this work.

\section{References}

1. UNODC. World Drug Report. Vienna, Austria: United Nations; 2008.

2. Anthony JC, Warner LA, Kessler RC. Comparative epidemiology of dependence on tobacco, alcohol, controlled substances and inhalants: basic findings from the National Comorbidity Survey. Exp Clin Psychopharmacol. 1994;2:244-268

3. Copeland J, Swift W. Cannabis use disorder: epidemiology and management. Int Rev Psychiatry. 2009;21(2):96-103.

4. Budney AJ, Moore BA, Vandrey RG, Hughes JR. The time course and significance of cannabis withdrawal. J Abnorm Psychol. 2003;112(3):393-402.

5. Stephens RS, Roffman RA, Curtin L. Comparison of extended versus brief treatments for marijuana use. J Consult Clin Psychol. 2000;68(5):898-908.

6. Chung T, Martin CS, Cornelius JR, Clark DB. Cannabis withdrawal predicts severity of cannabis involvement at 1-year follow-up among treated adolescents. Addiction. 2008;103(5):787-799.

7. Levin KH, Copersino ML, Heishman SJ, et al. Cannabis withdrawal symptoms in non-treatment-seeking adult cannabis smokers. Drug Alcohol Depend. 2010;111(1-2):120-127.

8. Hart CL. Increasing treatment options for cannabis dependence: a review of potential pharmacotherapies. Drug Alcohol Depend. 2005;80(2):147-159.

9. Haney M, Hart CL, Vosburg SK, et al. Marijuana withdrawal in humans: effects of oral THC or divalproex. Neuropsychopharmacology. 2004;29(1):158-170.

10. Winstock AR, Lea T, Copeland J. Lithium carbonate in the management of cannabis withdrawal in humans: an open-label study. J Psychopharmacol. 2009;23(1):84-93.

11. Haney M, Hart CL, Ward AS, Foltin RW. Nefazodone decreases anxiety during marijuana withdrawal in humans. Psychopharmacology (Berl). 2003;165(2):157-165.

12. Haney M, Ward AS, Comer SD, Hart CL, Foltin RW, Fischman MW. Bupropion SR worsens mood during marijuana withdrawal in humans. Psychopharmacology (Berl). 2001;155(2):171-179.

13. Budney AJ, Vandrey RG, Hughes JR, Moore BA, Bahrenburg B. Oral delta-9-tetrahydrocannabinol suppresses cannabis withdrawal symptoms. Drug Alcohol Depend. 2007;86(1):22-29.

14. Levin FR, Mariani JJ, Brooks DJ, Pavlicova M, Cheng W, Nunes EV. Dronabinol for the treatment of cannabis dependence: A randomized, double-blind, placebo-controlled trial. Drug Alcohol Depend. 2011;116(1-3):142-150.

15. Haney M, Hart CL, Vosburg SK, Comer SD, Reed SC, Foltin RW. Effects of THC and lofexidine in a human laboratory model of marijuana withdrawal and relapse. Psychopharmacology (Berl). 2008;197(1):157-168.
16. Mauri MC, Volonteri LS, Fiorentini A, Pirola R, Bareggi SR. Two weeks' quetiapine treatment for schizophrenia, drug-induced psychosis and borderline personality disorder: a naturalistic study with drug plasma levels. Expert Opin Pharmacother. 2007;8(14):2207-2213.

17. West R, Gossop M. Overview: a comparison of withdrawal symptoms from different drug classes. Addiction. 1994;89(11):1483-1489.

18. Bressan RA, Crippa JA. The role of dopamine in reward and pleasure behaviour - review of data from preclinical research. Acta Psychiatr Scand Suppl. 2005(427):14-21.

19. Zhornitsky S, Rizkallah E, Pampoulova T, et al. Antipsychotic agents for the treatment of substance use disorders in patients with and without comorbid psychosis. J Clin Psychopharmacol. 2010;30(4):417-424.

20. Potvin S, Stip E, Roy JY. The effect of quetiapine on cannabis use in 8 psychosis patients with drug dependency. Can J Psychiatry. 2004;49(10):711.

21. Rizkallah E, Stip E, Zhornitsky S, et al. Clinical evolution of substance use disorder patients during treatment with quetiapine: a 12-week, open-label, naturalistic trial. Expert Opin Pharmacother. 2010;11(18):2947-2951.

22. sanofi-aventis. Neulactil Data Sheet. August 22, 2011. Available from: www.sanofi-aventis.com.au/products/nzl_ds_neulactil.pdf. Accessed on February 13, 2012.

23. Gossop M, Darke S, Griffiths P, et al. The Severity of Dependence Scale (SDS): psychometric properties of the SDS in English and Australian samples of heroin, cocaine and amphetamine users. Addiction. 1995;90(5):607-614.

24. Bastien $\mathrm{CH}$, Vallieres A, Morin CM. Validation of the Insomnia Severity Index as an outcome measure for insomnia research. Sleep Med. 2001;2(4):297-307.

25. Lovibond P, Lovibond S. The structure of negative emotional states: Comparison of the Depression Anxiety Stress Scales (DASS) with the Beck Depression and Anxiety Inventories. Behav Res Ther. 1995;33(3):335-343.

26. Swift W, Copeland J, Hall W. Choosing a diagnostic cut-off for cannabis dependence. Addiction. 1998;93(11):1681-1692.

27. Copeland J, Swift W, Roffman R, Stephens R. A randomized controlled trial of brief cognitive-behavioral interventions for cannabis use disorder. J Subst Abuse Treat. 2001;21(2):55-64; discussion 65-56.

28. Lee KS, Clough AR, Jaragba MJ, Conigrave KM, Patton GC. Heavy cannabis use and depressive symptoms in three Aboriginal communities in Arnhem Land, Northern Territory. Med J Aust. 2008;188(10):605-608.

29. Yang M, Charles M, Schaefer K, Wallenstein G. Interpreting score differences in the Insomnia Severity Index: using health-related outcomes to define the minimally important difference. Curr Med Res Opin. 2009;25(10):2487-2494.

30. Weinstein AM, Gorelick DA. Pharmacological treatment of cannabis dependence. Curr Pharm Des. 2011;17(14):1351-1358.

31. Haney M. The marijuana withdrawal syndrome: diagnosis and treatment. Curr Psychiatry Rep. 2005;7(5):360-366.

32. Colagiuri B, Morley K, Boakes R, Haber P. Expectancy in doubleblind placebo-controlled trials: an example from alcohol dependence. Psychother Psychosom. 2009;78(3):167-171.
Substance Abuse and Rehabilitation is an international, peer-reviewed, open access journal publishing original research, case reports, editorials, reviews and commentaries on all areas of addiction and substance abuse and options for treatment and rehabilitation. The manuscript management system is completely online and includes a very quick and fair

\section{Dovepress}

Submit your manuscript here: http://www.dovepress.com/substance abuse-and-rehabilitation-journal 